\title{
Who cares for the carers?
}

\author{
L. D'Cruz'
}

IN BRIEF

- Recognise the impact complaints have on dentists and their teams.

- Raise awareness of the need to establish systems to support the recipients of patients complaints.

- Place these problems in the context of the new NHS complaints procedure which is focused around the needs of the complainant.

The NHS complaints system has been overhauled again in England with the stated aim of simplifying a 'process' driven system and to give 'organisations greater flexibility to respond and encourage a culture that seeks and then uses people's experiences of care to improve quality.'. The complaints system that ran from 1996 to 31 March 2009 was considered 'too prescriptive and inflexible and not meeting the needs of the person making the complaint.'. Many fine words and hours of consultations have been devoted to analysing the problems with the complaints systems and the advantages of producing a new one that enables health services to listen to the patients, respond to their concerns and improve the services delivered. This patient-centred focus is the new rally cry in the modern twenty-first century NHS. Very little thought, however, appears to have been given to those against whom the complaints are invariably directed: the dentist and their teams. What will the new reforms, with one less layer of complaints management (the Healthcare Commission) do to the dynamics of complaints management? What effects on the dentist and their teams will come about as a result of the primary care trust's ability to investigate the complaint themselves if invited to do so by the patient?

What is the real personal effect of the complaint on a dentist and who looks after their emotional needs?

In customer care and management literature the complaint is looked upon as a welcome opportunity to review and improve services. For many clinicians, however, the experience of receiving a complaint is a profoundly negative one. In a study conducted amongst a group of general medical practitioners, ${ }^{3}$ the clinicians described their experiences of patients' complaints in three stages: initial impact, conflict and resolution. The first stage described being out of control, feelings of shock and panic and a sense of indignation towards patients generally. The second stage described the many conflicts generated by the complaint: emotional conflicts such as feelings of anger, depression and even suicide, conflicts around aspects

${ }^{1}$ General Dental Practitioner, PCT Dental Practice Advisor and Dento-legal Advisor, Woodford Green, Essex Correspondence to: Mr Len D'Cruz

Email:len.d'cruz@mps.org.uk

\section{Refereed Paper}

Accepted 27 March 2009

DOI: 10.1038/sj.bdj.2009.557

${ }^{\circ}$ British Dental Journal 2009; 207: 11-12 of professional identity including doubts about clinical competence, conflicts with family and colleagues and conflicts arising from the management of the complaint. The third stage described resolution. For many this meant practising defensively, for others it meant planning to leave general practice and for a minority no resolution was achieved. Some described how they became immune to complaints and a small minority described the complaint as a learning experience.

\section{No immunity from emotion}

Being a professional does not confer immunity from an emotional response to a complaint. It might mean that any verbal or written communication will be appropriately worded and objective, preferably with assistance from a third party, but there should be no underestimation of the impact a complaint may have or its lasting effects, no matter how trivial the complaint might appear to be.

Generally speaking, health care staff give more of themselves emotionally than other workers in service industries and are therefore much more vulnerable to criticism. Dentists particularly are working in very close proximity to their patients, often themselves stressed or anxious if the patient is nervous or during painful and uncomfortable procedures. No dental professional sets out to do harm or cause problems for patients yet it appears that when something goes wrong blame must always find a resting place and inevitability it falls to the treating clinician. 'There is a form of subculture growing that if the slightest thing goes wrong, and it may not be negligence, then a patient will run off to a solicitor and sue the hospital.'4

For an increasing number of dentists the complaint they received most recently was the first they had received for many years or ever. This makes it all the more difficult to cope with and reflects the consumerist nature of society and the supermarket style no quibbles guarantee expected to be thrown in with every course of treatment.

There are short term immediate effects on receiving a complaint, relating to self doubt and self confidence with the result there is a reduced ability to work confidently and decisively. ${ }^{5}$ This goes beyond the original complaint and clouds interactions with other patients. In dentistry 
where much of the work is of a similar nature, a lack of confidence may affect a significant proportion of the practitioner's work if, for example, the complaint relates to the diagnosis of caries or the performance of root canal therapy. The long term consequences are also significant as they may result in burn out ${ }^{6}$ and increased stress levels with 54\% indicating that fear of complaints and litigation contributed to stress at work. ${ }^{7}$

There is no doubt that complaints are on the increase and dentists and their teams must embrace this fact since it is common currency in the world of the consumer. The new complaints procedure offers an opportunity to establish a support network for the person who is complained about.

The regulations require that a 'responsible person'8 is designated by the practice. This person has to ensure compliance with complaints arrangements and to ensure that action is taken if necessary in the light of the outcome of a complaint. The responsible person will need to be a director, the sole proprietor or a partner in the practice and will also be responsible for signing off the definitive response that goes to the complainant. This responsible person is also uniquely positioned to ensure that the person against whom the complaint is directed gets as much support as possible to help deal with the emotional consequences of receiving a complaint.

The various response stages described by Jain and Ogden equate to the five stages of grief model described by Kübler-Ross ${ }^{9}$ of denial, anger, bargaining, depression and acceptance. The 'responsible person' can help to manage these responses by reassurance, support, understanding and empathy. Dentists value the support of their peers more than anything at these times and it often falls to advisers at defence organisations to fulfil this role. Accompanying these complaints is also an acute paranoia: will the rest of my patients find out? What will my colleagues think of me? Will this go to the GDC? Will I get struck off? Experienced colleagues will be able to put the complaint into context and proportion.

It behoves us as a profession to accept that complaints are an essential part of improving the quality of care we deliver but we should also be acutely aware of the impact these complaints have on the individuals who are the subject of these complaints, whether they are justified or not, and to provide the pastoral care to support them and prevent any long term emotional or health consequences as a result.

1. Department of Health. Listening, responding, improving - a guide to better customer care. Gateway Reference 11215 February 2009.

2. Department of Health. Making experiences count response to consultation. Gateway reference 9353 February 2008.

3. Jain A, Ogden J. General practitioners' experiences of patients complaints: qualitative study. Br Med J 1999; 318: 1596-1599.

4. Patients used to thank doctors ... now they sue them. South Wales Echo, 26 October 2006.

5. Cunningham W, Dovey $S$. The effect on medical practice of disciplinary complaints: potentially negative for patient care. N Z Med J 2000; 113: 454-455.

6. Denton D A, Newton J T, Bower E J. Occupational burnout and work engagement: a national survey of dentists in the United Kingdom. Br Dent J 2008; 205: E13. .

7. Kay E J, Lowe J C. A survey of stress levels, selfperceived health and health related behaviours of UK dental practitioners in 2005. Br Dent J 2008; 204: E19.

8. Local Authority Social Services and National Health Service Complaints (England) Regulations 2009 Para 4.

9. Kübler-Ross E. On death and dying. 1969. 